\title{
Waves Generated by Moving Pressure Disturbances in Rectangular and Trapezoidal Channels
}

\author{
Philip L.-F. Liu and Tso-Ren Wu \\ School of Civil and Environmental Engineering \\ Cornell University, Ithaca, NY 14853
}

\section{Introduction}

It is well known that in modeling the generation and propagation of ship waves the moving vessel can be modeled as a moving free surface pressure field. Since the ship waves might consist of both long wave and short wave components, the depth integrated long wave equations, such as the Green-Naghdi (GN) equations and the forced $\mathrm{KdV}(\mathrm{fKdV})$ equation might not be suitable for computing short wave components. Furthermore, the $\mathrm{fKdV}$ equation can only be applied to uni-directional wave propagation problems. For small Froude number flows, the fKdV equation approach is not valid. Although the Boundary Integral Equation Method (BIEM) approach, based on the Laplace equation and exact free surface boundary conditions, has been proposed (e.g., Cao et al., 1993), test cases are usually limited to the two-dimensional (vertical plane) cases. Because of the heavy computational demand, actual threedimensional BIEM realizations are still not practical.

In this paper, we shall focus our investigation on waves generated by a moving pressure disturbance in a straight channel. Although the model presented here allows an arbitrary channel crosssection, only the results for rectangular and trapezoidal cross-section are discussed. The model equations are capable of modeling a wide range of wave components, $0 \leq k h \leq \pi$, where $k$ is the wave number and $h$ the water depth. It is well known that for the rectangular channel, a runaway solitary wave can be generated by a moving ship if the Froude number, $F r=U / \sqrt{g h}$, with $U$ being the speed of the moving pressure field and $g$ the gravitational acceleration, is near unity. In this paper, we shall investigate the effects of the slope of sidewalls on the characteristics of the runaway solitary waves, if any.

\section{Model equations and numerical scheme}

The depth-integrated model equations for describing fully nonlinear and weakly dispersive waves have been presented by many researchers. In these models the atmospheric pressure is always assumed to be a constant. Therefore, these models cannot be used to investigate the wave generation and propagation induced by moving pressure disturbances. To include the atmospheric pressure variation in the existing models is straightforward: The only term needs to be added is the free surface pressure gradient in the momentum equation. Thus, the resulting model equations take the following form:

$$
\begin{aligned}
& \frac{\partial \zeta}{\partial t}+\nabla \cdot\left[(\varepsilon \zeta+h) \mathbf{u}_{\alpha}\right]+\mu^{2} \nabla \cdot\left\{\left(\frac{z_{\alpha}{ }^{2}}{2}-\frac{h^{2}}{6}\right) h \nabla\left(\nabla \cdot \mathbf{u}_{\alpha}\right)+\left(z_{\alpha}+\frac{h}{2}\right) h \nabla\left[\nabla \cdot\left(h \mathbf{u}_{\alpha}\right)\right]\right\} \\
& +\varepsilon \mu^{2} \nabla \cdot\left\{\zeta\left[\left(z_{\alpha}-\frac{1}{2} \varepsilon \zeta\right) \nabla\left(\nabla \cdot\left(h \mathbf{u}_{\alpha}\right)\right)+\frac{1}{2}\left(z_{\alpha}{ }^{2}-\frac{1}{3} \varepsilon^{2} \zeta^{2}\right) \nabla\left(\nabla \cdot \mathbf{u}_{\alpha}\right)\right]\right\}=O\left(\mu^{4}\right)
\end{aligned}
$$




$$
\begin{aligned}
& \frac{\partial \mathbf{u}_{\alpha}}{\partial t}+\nabla \zeta+\frac{1}{2} \varepsilon \nabla\left|\mathbf{u}_{\alpha}\right|^{2}+\nabla p+\mu^{2}\left\{\nabla\left[z_{\alpha}\left(\nabla \cdot\left(h \frac{\partial \mathbf{u}_{\alpha}}{\partial t}\right)\right)\right]+\nabla\left[\frac{1}{2} z_{\alpha}^{2}\left(\nabla \cdot \frac{\partial \mathbf{u}_{\alpha}}{\partial t}\right)\right]+\nabla\left[\frac{\partial \mathbf{u}_{\alpha}}{\partial t}\left(\nabla \cdot\left(h \mathbf{u}_{\alpha}\right)+z_{\alpha} \nabla \cdot \mathbf{u}_{\alpha}\right)\right]\right\} \\
& +\varepsilon \mu^{2} \nabla\left\{\frac{1}{2} z_{\alpha}^{2} \mathbf{u}_{\alpha} \cdot \nabla\left(\nabla \cdot \mathbf{u}_{\alpha}\right)+z_{\alpha} \mathbf{u}_{\alpha} \cdot \nabla\left(\nabla \cdot h \mathbf{u}_{\alpha}\right)+\frac{1}{2}\left[\nabla \cdot\left(h \mathbf{u}_{\alpha}\right)\right]^{2}-\zeta \nabla \cdot\left(h \frac{\partial \mathbf{u}_{\alpha}}{\partial t}\right)\right\} \\
& +\varepsilon^{2} \mu^{2} \nabla\left\{\zeta\left(\nabla \cdot h \mathbf{u}_{\alpha}\right)\left(\nabla \cdot \mathbf{u}_{\alpha}\right)-\frac{1}{2} \zeta^{2} \nabla \cdot\left(\frac{\partial \mathbf{u}_{\alpha}}{\partial t}\right)-\zeta \mathbf{u}_{\alpha} \cdot \nabla\left(\nabla \cdot h \mathbf{u}_{\alpha}\right)\right\} \\
& +\varepsilon^{3} \mu^{2} \nabla\left\{\frac{1}{2} \zeta^{2}\left(\nabla \cdot \mathbf{u}_{\alpha}\right)^{2}-\frac{1}{2} \zeta^{2} \mathbf{u}_{\alpha} \cdot \nabla\left(\nabla \cdot \mathbf{u}_{\alpha}\right)\right\}=O\left(\mu^{4}\right)
\end{aligned}
$$

in which $\zeta$ denotes the free surface elevation, and $\mathbf{u}_{\alpha}=(u, v)$ denotes the horizontal velocity vector evaluated at $z=z_{\alpha}$ and $p$ the atmospheric pressure. Equation (1) is the depth integrated continuity equation, while (2) is the horizontal momentum equation. In deriving these model equations, the characteristic water depth, $h_{0}$, is assumed to be small in comparison with the horizontal length scale, $\lambda$, i.e., $\mu^{2}=\left(h_{0} / \lambda\right)^{2} \ll 1$. The nonlinearity is assumed to be $O(1)$, i.e., $\varepsilon=a / h_{0}=O(1)$. Here, $a$ represents the characteristic wave amplitude. The accuracy of the model equations given in (1) and (2) are up to $O$ $\left(\mu^{2}\right)$. It is well known that if $z_{\alpha}$ value is properly chosen, the resulting model equations can be used to model a wide range of wave number components. For instance, if $z_{\alpha}=-0.531 \mathrm{~h}$ is employed, the model equations can provide adequate description of wave propagation in terms of phase speed and group velocity for $0 \leq k h \leq \pi$.

A high order numerical scheme utilizes the fourth order predictor-corrector time-integration scheme is used in solving the model equations. The fourth order finite differencing is also used in evaluating the spatial derivatives. Similar schemes have been successfully used in modeling surface wave phenomena.

\section{Verifications of numerical model}

As a first step to validate the numerical model, 1D examples for waves generated in a channel with rectangular cross-section are investigated. Present solutions are compared with those obtained from the forced $\mathrm{KdV}$ equations and BIEM based on the Laplace equation (Cao et al., 1993). The moving pressure region has a length $L$ and is moving with a speed $U=F r C_{0}$, where $F r$ is the Froude number, $C_{0}=\sqrt{g h}$ is the critical phase speed, and $h$ is the water depth. The pressure varies as a cosine function in the region with the maximum amplitude $P_{m}$. In this example, $F r=1$ and $P_{m}=0.1$ are used. The first comparison is made between solutions obtained from the BIEM model (Cao et al., 1993) and the present model. Since the BIEM solves the potential flow exactly, its solutions can be used to validate other approximate models. In Figure 1, four snap shots of the free surface profiles are compared. The agreement is very good. To examine the importance of nonlinear effects, present numerical results are also compared to those of $\mathrm{fKdV}$ equation provided by Cao et al. (1993). We reiterate here that the fKdV model is valid for $O(\varepsilon)=O\left(\mu^{2}\right) \ll 1$. As shown in Figure 2, the numerical solutions obtained from the fKdV and the present model are quite different. The nonlinearity in this problem is very strong; using the leading wave amplitude as an estimate, $\varepsilon=a / h \approx 0.5$. Therefore, the $\mathrm{fKdV}$ model, which requires that $\varepsilon \ll 1$, predicts much slower (and incorrect) phase speed and is no longer valid. These comparisons indicate that the present model can account for the effects of nonlinearity and dispersive accurately. 


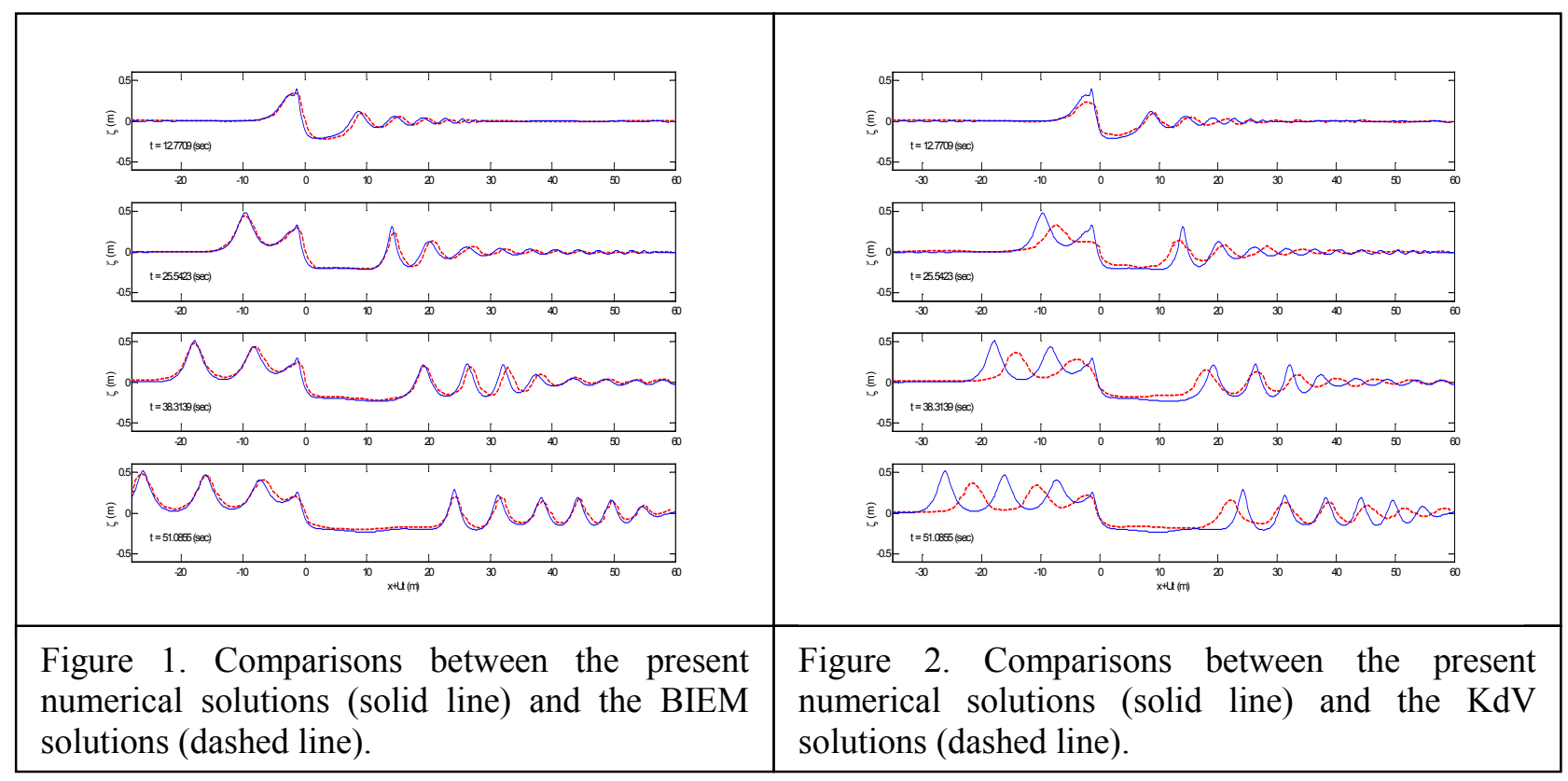

\section{Waves generated in rectangular and trapezoidal channels}

Based on the 1-D simulations, the fFNBM has been validated for the wave generation by a moving surface pressure in a rectangular channel. Further simulations will be applied to trapezoidal channels. The additional complexity of simulating waves in a trapezoidal channel is the appearance of the sloping sidewalls. Hence the generated waves will run up and down the sloping banks. A numerical method has been developed to treat the moving shoreline.

To characterize the flow in a trapezoidal channel, the Froude number is defined as $F r=\sqrt{g D}$, where $D=A / T$ and $A$ is the channel cross sectional area and $T$ is the surface width of the channel. Furthermore, the pressure is also normalized by $\rho g D$, i.e., $p=P_{m} \rho g D$. A series of numerical experiments has been carried out by using the same surface pressure field as the $1 \mathrm{D}$ case with $P_{m}=0.05$. The moving pressure region is $4 \mathrm{D}$ by $8 \mathrm{D}$. In the experiments, the surface width $T=40 D$ and both $A$ and $D$ are kept constants. Several sidewall slopes are examined: $s=\infty$ (vertical wall and rectangular channel), 1, 0.2 and 0.1 (triangular channel). We remark here that the triangular channel has the largest maximum water depth and the rectangular channel the smallest maximum depth. For each channel configuration, three moving pressure speeds are generated, resulting in three Froude numbers: $F_{r}=0.8,0.9$ and 1.0. However, numerical results shown here are only for the case of $F_{r}=0.8$. In Figure 3, snap shots of the free surface elevations in front of the moving pressure field are shown for four different sidewall slopes. For a steep sidewall slope, including the rectangular channel, the leading waves are moving faster than the moving pressure, and they tend to form run-away solitons. The sidewall slope causes waves to first refract towards the sidewall, and then be reflected back. For a milder sidewall slope, this process makes it difficult to form a straight crest line. In figure 3, the trailing wave patterns are also shown. The transverse waves are obvious in four different slopping sidewalls. However, the diverging waves are difficult to observe in the case of slope $=1 / 9.9$. 


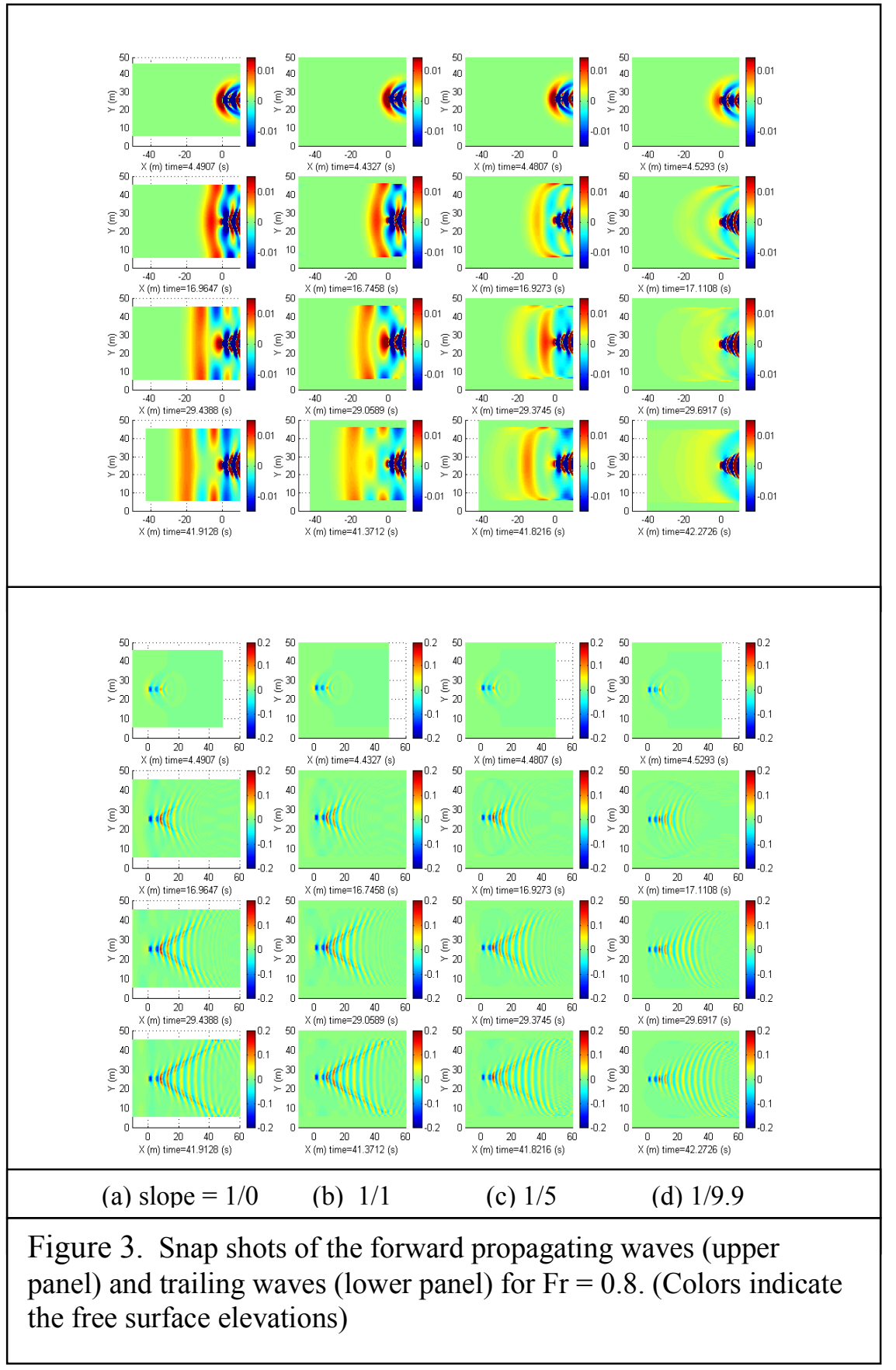

\section{References}

Cao, Y., Beck, R. F., and William, W. S. (1993). 'Numerical computations of two-dimensional solitary waves generated by moving disturbances', Int. J. Numer. Methods Fluids, 17, 905-920. 


\section{Discussion Sheet}

\begin{tabular}{|c|c|c|c|c|}
\hline Abstract Title : & \multicolumn{4}{|c|}{$\begin{array}{l}\text { Waves generated by moving pressure distribances in rectangular and } \\
\text { trapezoidal channels }\end{array}$} \\
\hline \multicolumn{2}{|c|}{ (Or) Proceedings Paper No. : } & 29 & Page: & 111 \\
\hline First Author : & \multicolumn{4}{|c|}{ Liu, P. L-F } \\
\hline Discusser : & \multicolumn{4}{|c|}{ D. Howell Peregrine } \\
\hline \multicolumn{5}{|c|}{$\begin{array}{l}\text { In my experiments on solitary waves propagating along trapezoidal channels } \\
\text { (Peregrine, } 1969 \mathrm{JFM} \text { Vol.35) I found that for slopes } 30^{\circ} \text { or less it was very difficult } \\
\text { to avoid wave breaking. Please comment. }\end{array}$} \\
\hline \multicolumn{5}{|c|}{$\begin{array}{l}\text { Yes, we also observe numerically that waves will "break" when the slope is mild. } \\
\text { Presently, we use a simple "wave breaking" model to treat the breaking waves. } \\
\text { However, the wave breaking model is based on the information for normal incident } \\
\text { waves. Further studies are necessary for determining the breaking wave condition for } \\
\text { oblique incident waves. }\end{array}$} \\
\hline
\end{tabular}




\section{Discussion Sheet}

\begin{tabular}{|c|c|c|c|c|}
\hline Abstract Title : & \multicolumn{4}{|c|}{$\begin{array}{l}\text { Waves generated by moving pressure distribances in rectangular and } \\
\text { trapezoidal channels }\end{array}$} \\
\hline \multicolumn{2}{|c|}{ (Or) Proceedings Paper No. : } & 29 & Page: & 111 \\
\hline First Author : & \multicolumn{4}{|c|}{ Liu, P. L-F } \\
\hline Discusser : & \multicolumn{4}{|c|}{ Paul D. Sclavounos } \\
\hline \multicolumn{5}{|c|}{ Questions / Comments : } \\
\hline \multicolumn{5}{|c|}{$\begin{array}{l}\text { We have recently completed a paper where we looked into the generation of solitions } \\
\text { by an advancing pressure distribution in shallow water modeled after a } 3 \text {-D } \\
\text { Boussinesq equation. We found that for a domain of infinite transverse extent, run- } \\
\text { away solitions exist for depth Froude numbers of up to about } 1 \text {. For supercritical } \\
\text { Froude numbers we found that the solitary-like waves stay attached to the } \\
\text { disturbance. Have you observed the same when your side boundaries are far from the } \\
\text { disturbance? }\end{array}$} \\
\hline \multicolumn{5}{|l|}{$\begin{array}{l}\text { Author's Reply : } \\
\text { (If Available) }\end{array}$} \\
\hline \multicolumn{5}{|c|}{$\begin{array}{l}\text { No, not really. I thought that the run-away soliton with a straightline crest can only be } \\
\text { generated in a channel, since the side wall effects are important. I would like to read } \\
\text { your paper when it is available. }\end{array}$} \\
\hline
\end{tabular}

